\title{
Global/Local Processing in Autism: Not a Disability, but a Disinclination
}

\author{
Kami Koldewyn ${ }^{1}$, Yuhong Jiang ${ }^{2}$, Sarah Weigelt ${ }^{1}$, and Nancy Kanwisher ${ }^{1}$ \\ ${ }^{1}$ Department of Brain and Cognitive Science and McGovern Institute for Brain Research, \\ Massachusetts Institute of Technology, Cambridge, MA, USA \\ ${ }^{2}$ Department of Psychology, University of Minnesota, Minneapolis, MN, USA
}

\begin{abstract}
It is widely suggested that ASD is characterized by atypical local/global processing, but the published findings are contradictory. In an effort to resolve this question, we tested a large group of children on both a free-choice task and an instructed task using hierarchical local-global stimuli. We find that although children with autism showed a reduced preference to report global properties of a stimulus when given a choice, their ability to process global properties when instructed to do so is unimpaired. These findings support prior claims that people with ASD show a disinclination, not a disability, in global processing, and highlight the broader question of whether other characteristics of autism may also reflect disinclinations rather than disabilities.
\end{abstract}

\section{Keywords}

Autism Spectrum Disorder; Global/Local Processing; Global Attention; Cognitive Development

\section{Introduction}

Extensive research has investigated the hypothesis that people with autism differ from typical individuals in their processing of global versus local information, but the precise nature and even the existence of this putative difference remains unclear. Global information processing, which is the ability to integrate piecemeal information into a coherent whole - to grasp the "gist" - is critical in sensory processing, communication and social interaction (Navon 1983); Happe \& Frith 2006). We combine information about objects and their surroundings to understand scenes, words and their syntax to understand sentences and social cues and their context to understand interactions between people. Conversely, the ability to focus on and perceive the local details of an object or situation is also important, for example to locate one person in a crowd or to detect a fleeting but revealing emotional expression flashing across a friend's face. Successful interaction with the world requires

Corresponding Author: Kami Koldewyn, PhD., McGovern Institute for Brain Research, MIT, Building 46 Room 4141 (Kanwisher Lab), Cambridge, MA 02139, kamik@ mit.edu, phone: 617-258-0670, fax: 617-258-8654.

Kami Koldewyn is at Massachusetts Institute of Technology in the McGovern Center for Brain Research and the Department of Brain and Cognitive Sciences in Cambridge Massachusetts, USA. Sarah Weigelt was also at Massachusetts Institute of Technology in the McGovern Center for Brain Research and the Department of Brain and Cognitive Sciences in Cambridge Massachusetts, USA. Nancy Kanwisher is the Walter A. Rosenblith Professor of Cognitive Neuroscience in the Department of Brain and Cognitive Sciences and at the McGovern Center for Brain Research at Massachusetts Institute of Technology in Cambridge Massachusetts, USA. Yuhong Jiang is a professor in the Psychology department at the University of Minnesota in Minneapolis Minnesota, USA.

Sarah Weigelt is now at Westfälische Wilhelms-Universität Münster in the Institut für Psychologie in Münster, Germany.

Conflict of interest

The authors declare that they have no conflict of interest. 
both local and global processing because the behavioral goals of the moment can rely on either kind of information, or both. What exactly then is the difference, if any, in how people with ASD process local versus global information?

Three subtly different (and nonexclusive) hypotheses have been proposed about local/global processing in ASD. Individuals with ASD have been hypothesized to have i) impaired global processing, i.e., an actual deficit or disability in global processing (Behrmann et al. 2006; Frith and Happé 1994; Happé and Frith 1996; Happé and Booth 2008), ii) enhanced local processing (Mottron et al. 2006; Mottron et al. 2003; Plaisted et al. 2003), and/or iii) a bias or default preference to focus more on local than global information by default (Happé and Frith 2006; Plaisted et al. 1999). The evidence on each of these hypotheses is mixed.

Evidence for the impaired global processing hypothesis comes from studies that explicitly instruct subjects to report the global or local level of hierarchical stimuli (e.g. "report the large letter" for stimuli in which a set of small letters is arranged in the shape of a large letter). Several of these studies have found that both children and adults with ASD show reduced global precedence compared to typically developing groups (Behrmann et al. 2006; Rinehart et al. 2000; Wang et al. 2007). However, other studies using the same type of task have reported comparable performance between with ASD and typical controls, in both children and adults (Hayward et al. 2012; Iarocci et al. 2006; Ozonoff et al. 1994; Plaisted et al. 1999; Scherf et al. 2008). Studies that tested Gestalt grouping or other measures of global perception have also shown mixed results. Compared with typically developing individuals, adults and adolescents with ASD have shown better (Perreault et al. 2011), worse (Grinter 2010; Nakano 2010) and similar performance (Vandenbroucke et al. 2008) on a variety of cognitive tasks that rely on global processing. It is likely that discrepancies within these findings are partly due to differences in the age at which participants are tested (children vs. adult), phenotypic variability within ASD, and the wide range of stimuli used in these studies. Taken together, however, it remains unclear whether individuals with ASD are impaired in global processing, and Frith and Happe, who originally supported this view, now support the alternative "local preference" view discussed below.

According to the second hypothesis, people with ASD have superior abilities in low-level, local perceptual processing rather than deficits in global processing (for review see: Mottron et al. 2006). The "enhanced perceptual functioning" theory of autism holds that individuals with ASD are biased towards local processing but not impaired at global perception. This bias is not the result of "choice" but reflects a difference between ASD and typical controls in brain structure, organization, and connectivity (e.g., higher activity in perceptual areas of the brain, and/or increased short-range connectivity in "early" perceptual areas).

Accordingly, people with ASD would perform as well as typical controls on global tasks while performing better on tasks that require local attention or perception (Mottron et al. 2003; Plaisted et al. 2003). When the two levels of processing are in conflict, this hypothesis predicts that people with autism will show increased local interference during a global task (i.e., greater disruption of global processing from conflicting local information) while showing typical levels of global interference during a local task (i.e., greater disruption of local processing from conflicting global information). While some studies have indeed found increased local interference for global tasks in ASD (Behrmann et al. 2006; Wang et al. 2007), others have not (Hayward et al. 2012; Plaisted et al. 1999).

The third hypothesis, called here the "local preference" hypothesis, holds that any difference in local/global processing in ASD reflects neither impaired global processing nor enhanced perceptual functioning, but a default preference to process local information when given the choice (Happé and Frith 2006; Plaisted et al. 2003). A difference in preference or choice, in the absence of an actual disability, may have important consequences for development, by 
affecting the information attended and hence the learning that occurs. It can also have a real impact on adult cognition, since global information will be missed just as much if people choose not to attend to it as if they simply cannot process it. But the two are fundamentally different, with importantly different implications for remediation.

The available data on the third hypothesis are also mixed. Plaisted et al (Plaisted et al. 1999) found that children with ASD performed much like typically developing children in a "selective attention" task in which they were instructed to attend globally in one block of trials, and to attend locally in another block. However, in a "divided" attention task, in which participants were instructed to monitor both global and local levels, children with ASD performed better on the local targets whereas typically developing children performed better on the global targets. On the basis of these findings, Plaisted et al argue that children with ASD may voluntarily attend to the local level, unless instructed to do otherwise. Using a similar design but testing young adults, Hayward and colleagues (Hayward et al. 2012) replicated the finding of comparable performance between individuals with ASD and typical controls in the selective attention task, but --in contrast to Plaisted et al.-- found no evidence of a stronger local bias in the divided attention task for their ASD group. However, the divided attention task used in these two studies is not a pure measure of default attention. Subjects are instructed in this task to monitor both global and local levels, that is, respond to a target whether it is at the global or local level. A more direct test of default attentional preferences would allow the subject to freely choose to attend to and report either level without instruction about which level to attend to.

Here we attempted to address the inconsistencies in the literature by testing a large number of subjects on tasks that directly tap into instructed and default attention to local and global information in compound stimuli. Our study asked two questions: i) do individuals with ASD in fact process local and global information differently from typical individuals, and ii) do performance differences on local/global tasks reflect a difference in ability (i.e., what the subject is actually able to do when they try) or a difference in preference (what they elect to do when given a choice)?

To address these questions, we tested participants in two experiments that used hierarchical shapes such as a triangle made of squares (Navon 1983). In Experiment 1, we asked participants to categorize such hierarchical stimuli, but gave no indication whether each stimulus should be categorized at the local or global level. In Experiment 2, we measured participants' ability to process the same hierarchical stimuli at either the local or the global level. Experiment 1 allowed us to measure the default inclination of participants to attend to either the global or local level of a hierarchical stimulus whereas Experiment 2 allowed us to measure the ability of participants to attend to the global or local level when instructed. Importantly, the same stimuli were used in both experiments and the same participants were tested in both experiments in a single session, allowing us to assess whether atypical global/ local processing in individuals with ASD originates primarily from a fundamental disability or from a disinclination.

\section{Methods}

\section{Participants}

Participants were 45 typically developing children and 45 children with ASD aged 5-12 years ( 9 girls and 36 boys in each group). The ASD phenotype was carefully characterized, including both DSM-IV diagnosis by clinicians specializing in neurodevelopmental disorders and the administration of the ADOS by research-reliable psychologists. Three additional participants with ASD and two additional typical participants were recruited and completed testing but were later excluded from the data set because their accuracy across all 
conditions in Experiment 2 was less than 80\%. All participants had normal or corrected-tonormal visual acuity. Participants received modest monetary compensation and small motivating prizes for their participation. Because of the visual-spatial nature of our tasks, we matched our groups not only on age but also on non-verbal IQ, measured by the Kaufman Brief Intelligence Test. Characterization data for all participants is shown in table 1.

Children with ASD were recruited through the SFARI database and the Boston Autism Consortium. Typically developing children were recruited from the local community. Potential participants were excluded if they had any history of birth or brain trauma, noncorrected visual impairments or a non-verbal IQ of less than 80. Typically developing participants were further excluded if they scored higher than 11 on the Social Communication Questionnaire (see description below), had any developmental disorder or an immediate family history of ASD. Every participant signed an assent form and a parent or guardian signed an informed consent approved by the MIT Committee on the Use of Humans as Experimental Subjects.

Standardized Measures-All children were tested on a number of standardized tests. Data from all of these standardized tests are presented in Table 1.

1. Autism Diagnostic Observation Schedule (ADOS) (Lord et al. 2000): A structured observational assessment that provides opportunities for interaction and play while measuring social, communicative and repetitive behaviors that are diagnostic of ASD. Higher scores are indicative of greater autism symptom severity. When using the ADOS to differentiate between children with ASD and others, the sensitivity of the ADOS is .8 and the Specificity is .94 .

2. Social Communication Questionnaire (SCQ): A brief parent-report screening questionnaire to evaluate communication and social skills in people aged 4 years and above (Rutter et al., 2003). The SCQ was obtained from all participants. Higher scores indicate a greater concern that a child might have an ASD. Although a score of 15 is typically used in clinical settings to indicate concern that a child may have ASD, we used a more conservative cutoff score of 11 for the typically developing group. The SCQ has a reliability (Cronbach's alpha) of .89, a sensitivity of .86 and a specificity of .67 (distinguishing between those with ASD and others).

3. Social Responsiveness Scale (SRS) (Constantino and Gruber, 2005) is a parent report questionnaire used to assess the severity of social impairment in children with or without ASD. It is sensitive to subthreshold autistic symptoms in typically developing children. Higher scores on the SRS indicate a greater severity of social impairment. The reliability of the SRS is .9 Bolte 2008, its sensitivity is .78 and its specificity is .67.

4. The Kaufman Brief Intelligence Test II (k-bit) (Kaufman and Kaufman, 2004): The K-bit provides a short and reliable means of assessing intelligence in individuals aged 4-90. Only the nonverbal subtest was used, testing skills such as pattern recognition, analogy completion and mental rotation. In the age-range tested in this study (5-12), the reliability of the k-bit is .76 and its validity with other more detailed measures of IQ is high (e.g. the correlation of PIQ scores between the k-bit and the WAIS-III is .79).

5. Swanson, Nolan and Pelham Questionnaire, revised (SNAP-IV) (Bussing et al. 2008): A parent report where parents assess their child on symptoms of ADHD as defined by the DSM-IV (American, 1994). Higher scores on the SNAP-IV indicate a greater number of ADHD symptoms. Reliability on the SNAP-IV is .94, while its 
clinical validity (i.e. does scoring above cut-off on the SNAP-IV correlate with getting a diagnosis of ADHD) is .82.

6. Functional Acuity Contrast Test (F.A.C.T) (Ginsburg 1984): A chart designed to test visual acuity across 5 different spatial frequencies and 9 different contrasts. The chart is constructed of sine-wave gratings that can be oriented in three different ways (up, left, right). Participants indicate the orientation of each grating. The chart measures acuity by varying both the size of the grating (spatial frequency) and the contrast between the grating and the background. The F.A.C.T. test is standardly used, and is useful for not only testing general acuity but also identifying any differences in vision at specific spatial frequencies.

General Procedure-The experiments were programmed with psychtoolbox (Brainard 1997); Pelli 1997) implemented in MATLAB. Responses were made on a touch screen monitor (resolution: $1024 \times 769$ pixels, refresh rate: $60 \mathrm{~Hz}$ ). Experiment one and two were administered in a single session.

Experiment One-This experiment was designed to test children's default preference for attending to the global or local levels of a hierarchical stimulus. Experiment One was always completed before Experiment Two to ensure that children's free-choice responses were not influenced by previous experience with the stimuli or instruction about which level to attend to. There were two critical trials, preceded by three warm-up trials.

Warm-up trials: The first three trials served to establish the routine and to check that subjects understood the concept of the categorization task. On these trials, children were shown a sample object, such as a kitten, and asked to verbally state what the object was. After the verbal response, two picture choices were added to the bottom of the display, such as a rooster and a cat. Children were asked to touch the picture that went with the sample object (see Figure 1). The three trials involved a kitten (rooster/cat), the digit 3 (7/3), and a dog (flower, puppy). All children were $100 \%$ accurate in categorizing the sample objects on the warm-up trials, suggesting that they understood the nature of the task.

Critical Trials: The fourth and fifth trials consisted of hierarchical stimuli (Figure 1). On the first critical trial, the sample stimulus was a big triangle made of small squares. The two choices presented at the bottom of the screen matched the sample either at the global level (a big triangle made of small triangles) or at the local level (a big square made of small squares). Children were given no indication of whether the hierarchical stimulus should be matched based on the global level or the local level and were given no feedback. The second critical trial was similar, except that we used hierarchical letters. The sample was a big $\mathrm{S}$ made of small Hs, and the two choices were a big S made of small Ss (global match) and a big $\mathrm{H}$ made of small Hs (local match).

The warm-up and critical trials were run continuously without any interruptions. We used a minimum number of critical trials, as opposed to dozens (Rondan and Deruelle 2007) or hundreds (Plaisted et al. 2006), to get a clean measure of the child's default preference, untainted by any strategies that children are likely to adopt in longer tests (e.g., always categorizing at a specific level, or alternating between global and local responses).

Experiment Two-This experiment was designed to examine participants' ability to categorize hierarchical stimuli at either the global or the local level, by instructing them to attend to a specific level, either global or local (in different testing blocks). Success depended on the ability of participants to attend selectively to the instructed level. 
Stimuli were hierarchical shapes of triangles and squares (the same as the first critical trial in Experiment 1). Each child was tested in two task blocks (one global and one local), with task order counterbalanced across participants.

Trials were self-paced. During each trial, participants first touched a Go button at the bottom of the screen, upon which a stimulus was shown at the top of the screen. During the global block, participants were asked whether the big shape at the top of the screen was a "triangle" or a "square", and reported their answer by touching either the left ("triangle") or right ("square") response patch at the bottom of the screen. During the local block, participants were asked if the stimulus at the top of the screen was "made of" squares or triangles, again reporting their answer by touching either the triangle or the square at the bottom of the screen. The two response patches remained the same across the two blocks and illustrated congruent hierarchical objects (a triangle made of triangles, a square made of squares). Participants were asked to respond as quickly as possible while still choosing the correct answer. Figure 2 shows a sample trial.

The main experimental manipulations were i) task (report global or report local) and ii) stimulus condition (congruent, incongruent, or neutral). For congruent stimuli, local and global information were consistent (e.g., a triangle made of triangles). For incongruent stimuli, local and global information were inconsistent (e.g., a triangle made of squares). For neutral stimuli only the instructed level was present in the test stimulus (e.g., a big triangle in the global task, a small triangle in the local task). Because the only difference between the global and local tasks in the neutral condition was the size of the test stimulus, the neutral condition allowed us to control for the possibility that the local task might be more difficult simply because the size of the attended shapes were smaller than in the global task.

All participants completed two task blocks, each consisting of 36 trials that were evenly divided into the three congruency types: congruent, incongruent, or neutral. Trial order was random except for the first trial, which was always a neutral trial and was treated as a practice trial. Response accuracy and RT data was recorded for each trial. A correct response was followed by three rising tones. An incorrect response was followed by a prerecorded verbal correction that gave the correct response (e.g., "That was a big triangle").

\section{Results \\ Experiment One}

Averaged across the two critical trials, typical children were more likely than children with ASD to categorize hierarchical stimuli at the global level $(t(88)=2.34, p=0.02$; see Figure $3)$. This relationship held for each critical trial assessed individually $(p s<0.02)$. While typical children overwhelmingly preferred to categorize these stimuli at the global level, children with ASD showed no clear preference for either level (1-sample t-test vs. .5: $t(44)=$ $0.443, p=0.66$ ).

Importantly, this pattern of results is also consistent across the two trials. On average, participants did not change their response patterns between the two trials. The mean response (where $1=$ global and $0=$ local) on Trial 1 was .62 in the ASD group and .87 in the typical group. The mean response on Trial 2 was .62 in the ASD group and .84 in the typical group. Additionally, while onefifth of participants (20\% TD, 22\% ASD) produced inconsistent responses for the two test trials, the vast majority of participants produced consistent responses (both local, or both global). Response between the two trials was highly correlated $(r=.45, p<.001)$. Further analysis showed that the percentage of global and local responses differed substantially between ASD and TD children. More ASD children (27\%) 
categorized exclusively at the local level than did TD children (4\%), while more TD children (76\%) categorized exclusively at the global level than did ASD children (51\%).

Correlations with ADOS and SRS-Among children with ASD, local preference was significantly correlated with ADOS severity (total score on the ADOS, Spearman's rho = $0.374, p=0.04)$. In addition, across the entire sample of 90 children, local preference correlated significantly with lower social function as measured by the SRS ( $r h o=0.39, p=$ 0.001 ). Nonparametric tests of correlation (Spearman's rho) were used to assess these relationships because local preference scores were not normally distributed.

Correlations with Age or IQ-No correlations were found between local preference and either age or IQ for either typical children (age: $r h o=-0.161, p=0.29$; IQ: $r h o=-0.116, p=$ 0.45 ) or children with ASD (age: $r h o=0.046, p=0.76$; IQ: $r h o=0.014, p=0.93$ ), indicating that the slight and non-significant differences in age and IQ among the two groups cannot account for the observed differences in local/global preference between groups.

Visual Acuity-To test whether our results might be affected by differences in the perception of either low or high spatial frequencies between groups, we used the F.A.C.T. eye chart to assess participant's contrast sensitivity function across a wide range of spatial frequencies. These data were obtained from 38 of the typical children and 39 of the ASD children (see Table 2). In a repeated measures ANOVA with spatial frequency as a withinsubjects factor and group as a between subjects factor, we found a main effect of spatial frequency $(F(4,296)=77.142, p<0.001)$ but no main effect of group $(F(1,76)=0.399, p=$ $0.56)$ and no spatial frequency by group interaction $(F(4,296)=1.18, p=0.322)$. These results are consistent with recent reports that low-level visual processing is not affected in ASD (Koh et al. 2010; Tavassoli et al. 2011). Importantly, they suggest that the results from Experiment 1 cannot be accounted for by differences in spatial-frequency dependent contrast sensitivity between the two groups.

Is two trials enough? Our chief goal in this experiment was to test for group differences, which do not require test-retest reliability at the individual level. Indeed, even with just two trials per subject, we found a robust and significant difference between TD and ASD groups in this experiment. Further, even though it was not necessary for our research question, we do in fact find internal reliability in this study: the majority of both TD (80\%) and ASD (78\%) children responded consistently across the two trials (both local or both global), and responses between the two trials were significantly correlated. Further evidence from the reliability of this measure comes from the fact that it was significantly correlated with ADOS severity, and with lower social function as measured by the SRS. Thus, even with only two trials we are able to detect robust differences between groups, and reliable measures within individuals.

\section{Experiment Two}

Does the reduced tendency to report global information in ASD found in Experiment 1 reflect a disability or a disinclination to process global information? To answer this question, in Experiment 2, we measured the ability of children with ASD to categorize ambiguous stimuli at either the local or global level when instructed to do so. If children with ASD were less able to process stimuli at the global level, then they should perform disproportionately worse in the global task than the local task. In addition, they should struggle when reporting the global shape during incongruent trials (increased local interference), and should excel when reporting local shapes, even when the global shape is incongruent with the local shapes (decreased global interference). 
These predictions, however, were not borne out by the data. For the RT analysis, we excluded the first trial of each task block (it was treated as practice) as well as any incorrect trials. To reduce the influence of outliers, we used median rather than mean RT for each participant. There was a trend for RT in the global task to be faster than for the local task across groups (the main effect of task: $P(1,88)=3.675, p=0.058$ ), but there was no main effect of task in accuracy $(F(1,88)=0.137, p=0.71)$. Additionally, the ASD group was both slower $(F(1,88)=5.169, p=0.025)$ and less accurate $(F(1,88)=6.169, p=0.015)$ than the comparison group across all trials. Importantly, however, children with ASD were not disproportionately worse in the global versus local task compared with typical children: there was no interaction between task and group $(F(1,88)=0.181, p=0.672)$ in RT, and although this interaction was marginally significant in the accuracy data $(F(1,88)=3.6973$, $p=0.06$ ), this trend is in the reverse-to-predicted direction, with accuracy higher in the local task for the typical group than the ASD group (see Table 3).

Global vs. local interference-Both groups of children experienced interference from the task-irrelevant level, demonstrated by slower RTs and lower accuracy on incongruent trials compared with congruent or neutral trials. This congruency effect was the same for both global and local tasks (see Figure 4). In the RT data, the main effect of congruency was significant $F(2,176)=56.496, p<0.001$ but congruency did not interact with task, $F(2,176)$ $=0.176, p=0.842$, and there was no three-way interaction between congruency, task, and group $F(2,176)=0.287, p=0.751$. The $\mathrm{RT}$ results were echoed in the accuracy data (main effect of congruency: $F(2,176)=63.366, p<0.001$; congruency $\times$ task interaction: $F(2,176)$ $=0.093, p=0.911$; congruency $\times$ task $\times$ group interaction: $F(2,176)=0.093, p=0.91)$. In other words, there is no evidence that children with ASD showed increased local interference and/or decreased global interference, compared with typical children. Children with ASD do, however, show greater interference overall, resulting in a significant interaction between congruency and group in both $\mathrm{RT}(F(2,176)=8.139, p<0.001)$ and accuracy data $(F(2,176)=4.511, p=0.012)$.

The inclusion of neutral trials allowed us to test whether either RT or accuracy was influenced by the size of the stimuli. There was no significant difference in RT between the large neutral stimuli used in the global task and the small neutral stimuli used in the local task for either group (ASD: $t(44)=-1.60, p=0.12$; Typical: $t(44)=-1.34, p=0.19$ ) Likewise, neither group showed a significant effect of object size in the accuracy data (ASD: $t(44)=1.15, p=0.26$; Typical: $t(44)=-1.24, p=0.23$ ). Finally, in the global task, to perceive the global shape for a congruent stimulus it is necessary to group the small shapes. Grouping was not necessary to perceive the global shape of a neutral stimulus. The need for grouping (i.e., the difference between congruent and neutral trials in the global task) slowed down RT for the ASD group $(t(44)=2.95, p=0.005)$ and the typical comparison group $(t(44)=2.74, p=0.01)$, to the same degree (group by grouping interaction $F(1,64)=0.04, p$ $=0.84)$.

In the above analyses, we presented both accuracy and median RT data from correct trials, a standard measure of global/local processing of hierarchical stimuli. To ensure that our results were not specific to the use of median RT, we conducted statistical analyses using mean RT, filtering out outlier reaction times by removing trials that were more than $3 \mathrm{SD}$ above (or below) the mean reaction time for each individual for each condition. Our results remained largely the same except that the main effect of group was reduced to being marginally significant $(F(1,88)=3.208, p=0.08)$. All other statistical tests confirmed the analysis conducted using median RT values. Table 3 shows results from Experiment 2, including accuracy, mean RT, and median RT. 
Experiments 1 vs. 2-Did performance in Experiment 2 depend in any way on performance in Experiment 1? Here we focus on global interference (congruency effects measured in the local task) and local interference (congruency effects measured in the global task) ${ }^{1}$. We found no correlation for either group between participants' performance on the preference test from Experiment 1 (global preference score) and either global interference during the local task (ASD: $r h o=-0.065, p=0.72$; Typical: $r h o=-0.136, p=0.32$ ) or local interference during the global task (ASD: $r h o=-0.177, p=0.25$; Typical: $r h o=0.028, p=$ 0.86 ) on the ability test (global interference, local interference) in Experiment 2.

Although children with ASD showed no evidence of an imbalance between local and global processing in Experiment 2, they did suffer from greater interference when the two levels were incongruent, no matter which task was being performed. This is shown by the lack of a main effect of task $(F(1,88)=0.161, p=0.69)$ and no task by group interaction $(F(1,88)=$ $0.049, p=0.83)$ but a significant main effect of group $(F(1,88)=7.488, p=0.008)$ when RT interference was examined. To test whether greater overall interference effects in the ASD group could result from the well-known comorbidity of ADHD with autism, rather than autism itself, we measured the correlation between the degree of interference across both tasks and ADHD symptomotology in the ASD group.

Interference was not correlated with ADHD symptoms measured by the SNAP-IV ( $r=$ $-0.03, p=0.87)$. Interference also did not correlate with IQ in either group. Intriguingly, overall interference was mildly correlated with the total score on the ADOS $(r=0.369, p=$ 0.038 ) when IQ was controlled for. These results indicate that greater interference in the ASD group was not driven by comorbidity with ADHD or "general" problems in attentiveness, but may instead be related to autism symptomotology.

\section{Discussion}

When given a choice, children with ASD were less likely to report global information than typical children were, but when explicitly instructed to report global information they performed similarly to typical children. Indeed, children with ASD even showed strong interference from irrelevant global information in a local task, further indicating that global processing is intact in ASD. Thus, children with ASD have no impairment in processing global information, only a disinclination to report global information.

These findings are consistent with prior suggestions that differences in local/global processing in autism reflect differences in default preference, not differences in ability (for review, see: Happe \& Frith 2006), thus supporting the "local preference" hypothesis reviewed in the introduction. Our finding of similar global processing in ASD when participants are instructed to report global information is consistent with many prior studies in children (Plaisted et al. 1999; Scherf et al. 2008) and adults (Hayward et al. 2012), though see also (Behrmann et al. 2006), thus providing evidence against the "global deficit" hypothesis. Our finding of a reduced default preference for global information concurs with the two prior studies in children and adolescents that most explicitly discussed the "local preference" hypothesis (Mottron et al. 2003; Plaisted et al. 1999) but contrasts with another study using a similar method with adults (Hayward et al. 2012). However, these studies measured default preferences using a "divided attention" task, in which subjects were asked to monitor both global and local information. This task is not ideal for measuring default preferences, because it does not give the participant a choice of which level to attend to. In contrast, our default preference task was truly open-ended, enabling the subject to chose either global or local information. Further, we tested participants on only 2 trials as opposed

\footnotetext{
${ }^{1}$ Congruency effect, or interference, was measured as: (incongruent RT - congruent RT) / congruent RT.
} 
to dozens or hundreds of trials as in most previous studies (e.g. Wang et al. 2007)), which could have induced participants to develop strategies that mask their spontaneous preference. The acquisition of such implicit strategies depends on sensitivity to trial statistics and the amount of competition between implicit biases and explicit task instructions. As it has been previously shown that children with ASD differ from typical children in their reliance on implicit bias and their use of explicit strategies (Iarocci et al. 2006), using a paradigm that minimizes the influence of these factors may be important. While our use of a paradigm with only two trials limited our ability to look at inter-subject consistency, it gave us the ability to capture spontaneous choice. Finally, we tested a much larger number of participants with ASD than prior studies, giving us more power to either detect or reject group differences. For these reasons our study substantially strengthens prior evidence that individuals with ASD have weaker default preferences to process global information than do typical individuals, at least in childhood.

Why do children with ASD show reduced global preference, even though their ability to process local and global information is comparable to typical children? One possibility is that children with ASD may report the aspect of the stimulus they focused on first, without trying to determine the "correct" answer. In contrast, typical children may try to tailor their response to the inferred intent of the experimenter (Choe et al. 2005). One prior study used an approach similar to ours to look at default global/local biases in adults with ASD (Bolte et al. 2007). In five trials, participants were shown a hierarchical letter stimulus and then asked which letter they saw "first". Typical adults reported the global letter $84 \%$ of the time while those with ASD did so $70 \%$ of the time, a difference that did not reach statistical significance. The authors concluded that both typical adults and those with ASD show a preference for the global level of classification. However, Bolte et al.'s study showed a trend of the same between-group difference we found; a trend that may have reached significance if tested in a larger cohort of participants. It is also important to note that there is no "absolute" preference that can be determined from hierarchical stimuli. The proportion of global or local responses elicited by these stimuli depends greatly on the properties of the stimuli used in any particular study. More closely spaced local elements emphasize the global aspects of the stimuli and will increase the number of global responses (Kimchi 1992). For these reasons, one cannot take $50 \%$ as the "neutral" baseline. Instead, to assess local preference in ASD, the proper baseline comes from the typical comparison group. Thus, another reason that our results may have differed from the Bolte et al. study is that the stimuli used in their study were more densely packed than those in the current study, likely pushing responses towards the global level. Finally, they tested high-functioning adults with ASD, who may have learned that a global response is more likely to be what people are referring to in real world situations; in contrast, the children we tested may not yet have learned this.

Our findings may hold broader implications about the cognitive profile of children with ASD. Lacking a typically global processing style may cause significant problems when an individual with ASD interacts with others who automatically process and understand the "gist", even if it does not cause problems when he/she is told what aspect of a scene to focus on. It is important to remember, however, that the current study focused on perceptual global and local processing and cannot directly address how children with ASD might differ in their ability to "think globally" or the extent to which they focus on the details of ideas or abstractions. Our findings do, however, raise the possibility that other aspects of the cognitive profile in ASD may also reflect disinclinations rather than disabilities. A dichotomy between how people with ASD may perform in open-ended tasks and how they can perform with more specific instructions has been shown before in tasks as diverse as verbal semantics (Snowling 1986) and face perception (López 2010); See Happe \& Frith 2006 for a review of some of the relevant literature). The evidence reported here underlines 
the importance of this distinction between performance on open-ended versus highly structured tasks in ASD.

Aside from our evidence of intact global processing in the instructed task, Experiment 2 found an overall increase in interference from the task-irrelevant level in children with ASD. This finding provides some evidence contrary to the "enhanced perceptual functioning" hypothesis, which would have predicted increased local interference only during the global task in ASD. Because individuals with ASD have intact or superior visual search abilities (Baldassi et al. 2009; O'Riordan et al. 2001; Plaisted et al. 1998) this task-independent increase in interference is unlikely to reflect a deficit in selective attention. Instead, it suggests that simultaneous processing of two competing stimuli may differ in ASD, as has been suggested by a mathematical model of global/local processing of hierarchical stimuli in ASD (Johnson et al. 2010). This processing difference could reflect a possible increase in distractor interference or conflict control in ASD (Christ et al. 2011; Geurts et al. 2004; Kleinhans et al. 2005; Remington et al. 2009) but see (Keehn et al. 2010), a possibility worth investigating in future studies.

The idea that ASD is associated with atypical local/global processing has been a major theme in the ASD literature but its empirical support has been mixed. Our study substantially strengthens prior evidence that the ability to attend to local or global aspects of a stimulus is intact in children with ASD, who are simply less inclined to attend to and report global information. The consequences of this disinclination may be just as profound as those resulting from a true disability, but the options for treatment or remediation are quite different. The distinction between disabilities and disinclinations may be important for understanding other aspects of the cognitive phenotype of autism (Happé and Frith 2006), which may also turn out to reflect differences in inclination rather than ability.

\section{Acknowledgments}

The authors would especially like to thank all the participants and their families for their time and contribution to our research. We are grateful to all of the families at the participating SFARI Simplex Collection (SSC) sites, as well as the principal investigators (A. Beaudet, R. Bernier, J. Constantino, E. Cook, E. Fombonne, D. Geschwind, E. Hanson, D. Grice, A. Klin, R. Kochel, D. Ledbetter, C. Lord, C. Martin, D. Martin, R. Maxim, J. Miles, O. Ousley, K. Pelphrey, B. Peterson, J. Piggot, C. Saulnier, M. State, W. Stone, J. Sutcliffe, C. Walsh, Z. Warren, E. Wijsman). We are also grateful to all of the families participating in the Autism Consortium collection, as well as the principal investigators. This study was supported by funds from the Ellison Medical Foundation and the Simons Foundation.

\section{References}

Baldassi S, Pei F, Megna N, Recupero G, Viespoli M, Igliozzi R, Tancredi R, Muratori F, Cioni G. Search superiority in autism within, but not outside the crowding regime. Vision Research. 2009; 49(16):2151-2156. [PubMed: 19524608]

Behrmann M, Avidan G, Leonard GL, Kimchi R, Luna B, Humphreys K, Minshew N. Configural processing in autism and its relationship to face processing. Neuropsychologia. 2006; 44(1):110129. [PubMed: 15907952]

Bolte S, Holtmann M, Poustka F, Scheurich A, Schmidt L. Gestalt perception and local-global processing in high-functioning autism. Journal of Autism and Developmental Disorders. 2007; 8:1493-1504. 10.1007/s10803-006-0231-x. [PubMed: 17029017]

Brainard DH. The psychophysics toolbox. Spatial Vision. 1997; 10(4):433-436. [PubMed: 9176952]

Bussing R, Fernandez M, Harwood M, Wei Hou, Garvan CW, Eyberg SM, Swanson JM. Parent and teacher SNAP-IV ratings of attention deficit hyperactivity disorder symptoms: psychometric properties and normative ratings from a school district sample. Assessment. 2008; 15(3):317-328. [PubMed: 18310593] 
Choe KS, Keil FC, Bloom P. Children's understanding of the Ulysses conflict. Developmental Science. 2005; 8(5):387-392. [PubMed: 16048510]

Christ SE, Kester LE, Bodner KE, Miles JH. Evidence for selective inhibitory impairment in individuals with autism spectrum disorder. Neuropsychology. 2011; 25(6):690-701. [PubMed: 21728431]

Constantino, JN.; Gruber, CP. Social Responsiveness Scale (SRS). Los Angeles, CA: Western Psychological Services; 2005.

Frith U, Happé F. Autism: beyond "theory of mind". Cognition. 1994; 50(1-3):115-132. [PubMed: 8039356]

Geurts HM, Verté S, Oosterlaan J, Roeyers H, Sergeant JA. How specific are executive functioning deficits in attention deficit hyperactivity disorder and autism? Journal of Child Psychology and Psychiatry, and Allied Disciplines. 2004; 45(4):836-854.

Ginsburg AP. A new contrast sensitivity vision test chart. American Journal of Optometry and Physiological Optics. 1984; (6):403. [PubMed: 6742102]

Happé F, Frith U. The neuropsychology of autism. Brain : A Journal of Neurology. 1996; 119(Pt 4): 1377-1400. [PubMed: 8813299]

Happé F, Frith U. The weak coherence account: detail-focused cognitive style in autism spectrum disorders. Journal of Autism and Developmental Disorders. 2006; 36(1):5-25. [PubMed: 16450045]

Happé FG, Booth RD. The power of the positive: revisiting weak coherence in autism spectrum disorders. Quarterly Journal of Experimental Psychology (2006). 2008; 61(1):50-63. [PubMed: 18038338]

Hayward DA, Shore DI, Ristic J, Kovshoff H, Iarocci G, Mottron L, Burack JA. Flexible Visual Processing in Young Adults with Autism: The Effects of Implicit Learning on a Global-Local Task. Journal of Autism and Developmental Disorders. 2012; 42(11):2383-2392. [PubMed: 22391810]

Iarocci G, Burack JA, Shore DI, Mottron L, Enns JT. Global-local visual processing in high functioning children with autism: structural vs. implicit task biases. Journal of Autism and Developmental Disorders. 2006; 36(1):117-129. [PubMed: 16397823]

Johnson SA, Blaha LM, Houpt JW, Townsend JT. Systems factorial technology provides new insights on global--local information processing in autism spectrum disorders. Journal of Mathematical Psychology. 2010; 54(1):53-72. [PubMed: 23750050]

Kaufman, AS.; Kaufman, NL. Kaufman Brief Intelligence Test. Second Edition. Minneapolis, MN: NCS, Pearson, Inc.; 2004.

Keehn B, Lincoln AJ, Müller RA, Townsend J. Attentional networks in children and adolescents with autism spectrum disorder. Journal of Child Psychology and Psychiatry, and Allied Disciplines. 2010; 51(11):1251-1259.

Kleinhans N, Akshoomoff N, Delis DC. Executive functions in autism and Asperger's disorder: flexibility, fluency, and inhibition. Developmental Neuropsychology. 2005; 27(3):379-401. [PubMed: 15843103]

Koh HC, Milne E, Dobkins K. Spatial contrast sensitivity in adolescents with autism spectrum disorders. Journal of Autism and Developmental Disorders. 2010; 40(8):978-987. [PubMed: 20213250]

Lord C, Risi S, Lambrecht L, Cook EHJ, Leventhal BL, DiLavore PC, Pickles A, Rutter M. The autism diagnostic observation schedule-generic: a standard measure of social and communication deficits associated with the spectrum of autism. Journal of Autism and Developmental Disorders. 2000; 30(3):205-223. [PubMed: 11055457]

Mottron L, Burack JA, Iarocci G, Belleville S, Enns JT. Locally oriented perception with intact global processing among adolescents with high-functioning autism: evidence from multiple paradigms. Journal of Child Psychology and Psychiatry, and Allied Disciplines. 2003; 44(6):904-913.

Mottron L, Dawson M, Soulieres I, Hubert B, Burack J. Enhanced perceptual functioning in autism: an update, and eight principles of autistic perception. Journal of Autism and Developmental Disorders. 2006; 36(1):27-43. [PubMed: 16453071] 
Navon D. How many trees does it take to make a forest? Perception. 1983; 12(3):239-254. [PubMed: 6669451]

O'Riordan MA, Plaisted KC, Driver J, Baron-Cohen S. Superior visual search in autism. Journal of Experimental Psychology. Human Perception and Performance. 2001; 27(3):719-730. [PubMed: 11424657]

Ozonoff S, Strayer DL, McMahon WM, Filloux F. Executive function abilities in autism and Tourette syndrome: an information processing approach. Journal of Child Psychology and Psychiatry, and Allied Disciplines. 1994; 35(6):1015-1032.

Pelli DG. The VideoToolbox software for visual psychophysics: Transforming numbers into movies. Spatial Vision. 1997; 10(4):437-442. [PubMed: 9176953]

Perreault A, Gurnsey R, Dawson M, Mottron L, Bertone A. Increased sensitivity to mirror symmetry in autism. PloS One. 2011; 6(4):e19519. [PubMed: 21559337]

Plaisted K, Dobler V, Bell S, Davis G. The microgenesis of global perception in autism. Journal of Autism and Developmental Disorders. 2006; 36(1):107-116. [PubMed: 16450044]

Plaisted K, O'Riordan M, Baron-Cohen S. Enhanced visual search for a conjunctive target in autism: a research note. Journal of Child Psychology and Psychiatry, and Allied Disciplines. 1998; 39(5): 777-783.

Plaisted K, Saksida L, Alcantara J, Weisblatt E. Towards an understanding of the mechanisms of weak central coherence effects: experiments in visual configural learning and auditory perception. Philosophical Transactions of the Royal Society London: Biological Sciences. 2003; 358(1430): 375-386.

Plaisted K, Swettenham J, Rees L. Children with autism show local precedence in a divided attention task and global precedence in a selective attention task. Journal of Child Psychology and Psychiatry, and Allied Disciplines. 1999; 40(5):733-742.

Remington A, Swettenham J, Campbell R, Coleman M. Selective attention and perceptual load in autism spectrum disorder. Psychological Science. 2009; 20(11):1388-1393. [PubMed: 19843262]

Rinehart NJ, Bradshaw JL, Moss SA, Brereton AV, Tonge BJ. Atypical interference of local detail on global processing in high-functioning autism and Asperger's disorder. Journal of Child Psychology and Psychiatry, and Allied Disciplines. 2000; 41(6):769-778.

Rondan C, Deruelle C. Global and configural visual processing in adults with autism and Asperger syndrome. Research in Developmental Disabilities. 2007; 28(2):197-206. [PubMed: 16616454]

Rutter, M.; Bailey, A.; Lord, C. SCQ: Social Communication Questionnaire. Los Angeles, CA: Western Psychological Services; 2003.

Scherf KS, Luna B, Kimchi R, Minshew N, Behrmann M. Missing the big picture: impaired development of global shape processing in autism. Autism Research. 2008; 1(2):114-129. [PubMed: 19360658]

Tavassoli T, Latham K, Bach M, Dakin SC, Baron-Cohen S. Psychophysical measures of visual acuity in autism spectrum conditions. Vision Research. 2011; 51(15):1778-1780. [PubMed: 21704058]

Vandenbroucke MW, Scholte HS, van Engeland H, Lamme VA, Kemner C. Coherent versus component motion perception in autism spectrum disorder. Journal of Autism and Developmental Disorders. 2008; 38(5):941-949. [PubMed: 17952582]

Wang L, Mottron L, Peng D, Berthiaume C, Dawson M. Local bias and local-to-global interference without global deficit: a robust finding in autism under various conditions of attention, exposure time, and visual angle. Cognitive Neuropsychology. 2007; 24(5):550-574. [PubMed: 18416507] 

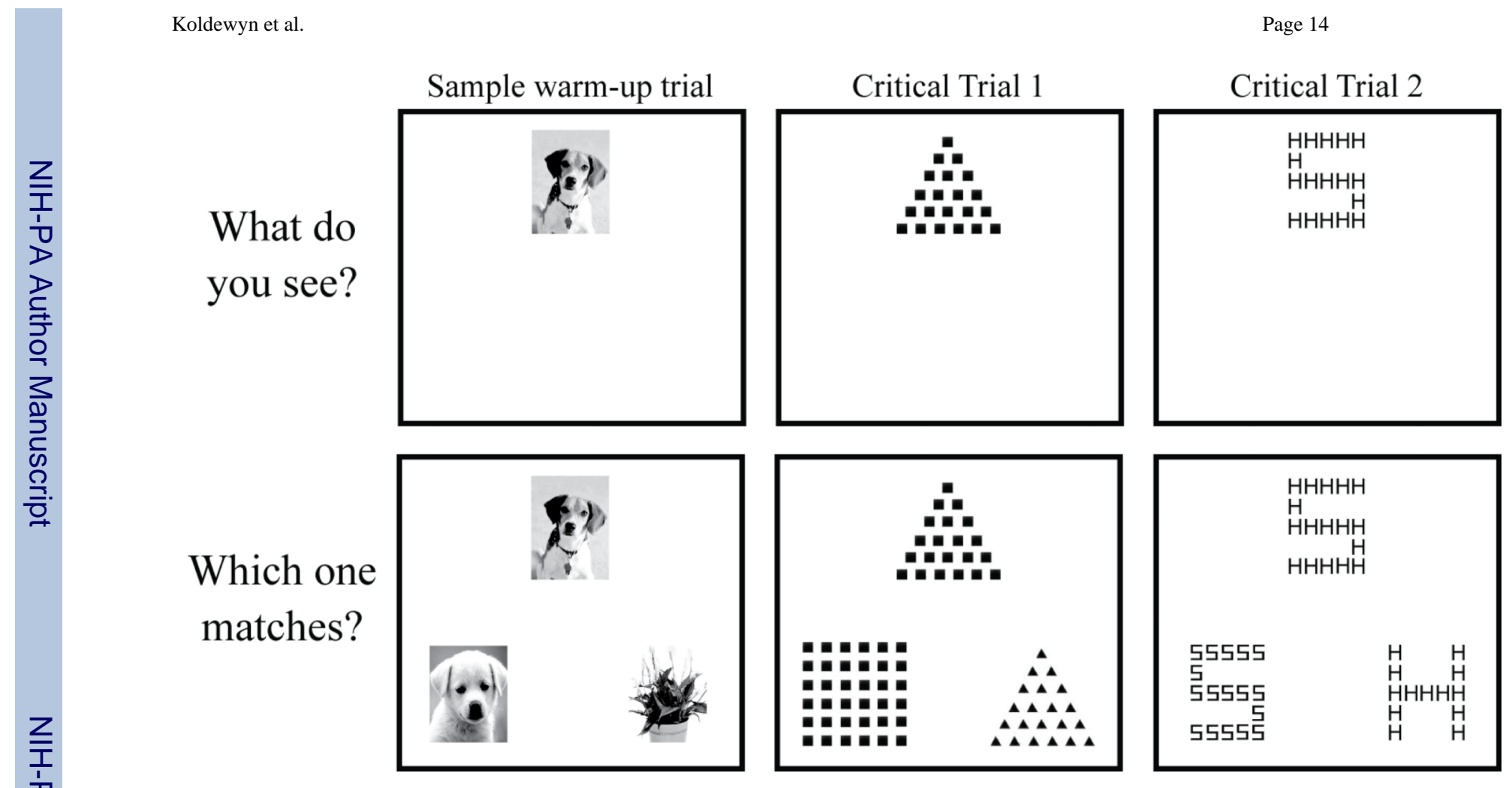

Figure 1.

Depiction of stimuli for Experiment 1. 


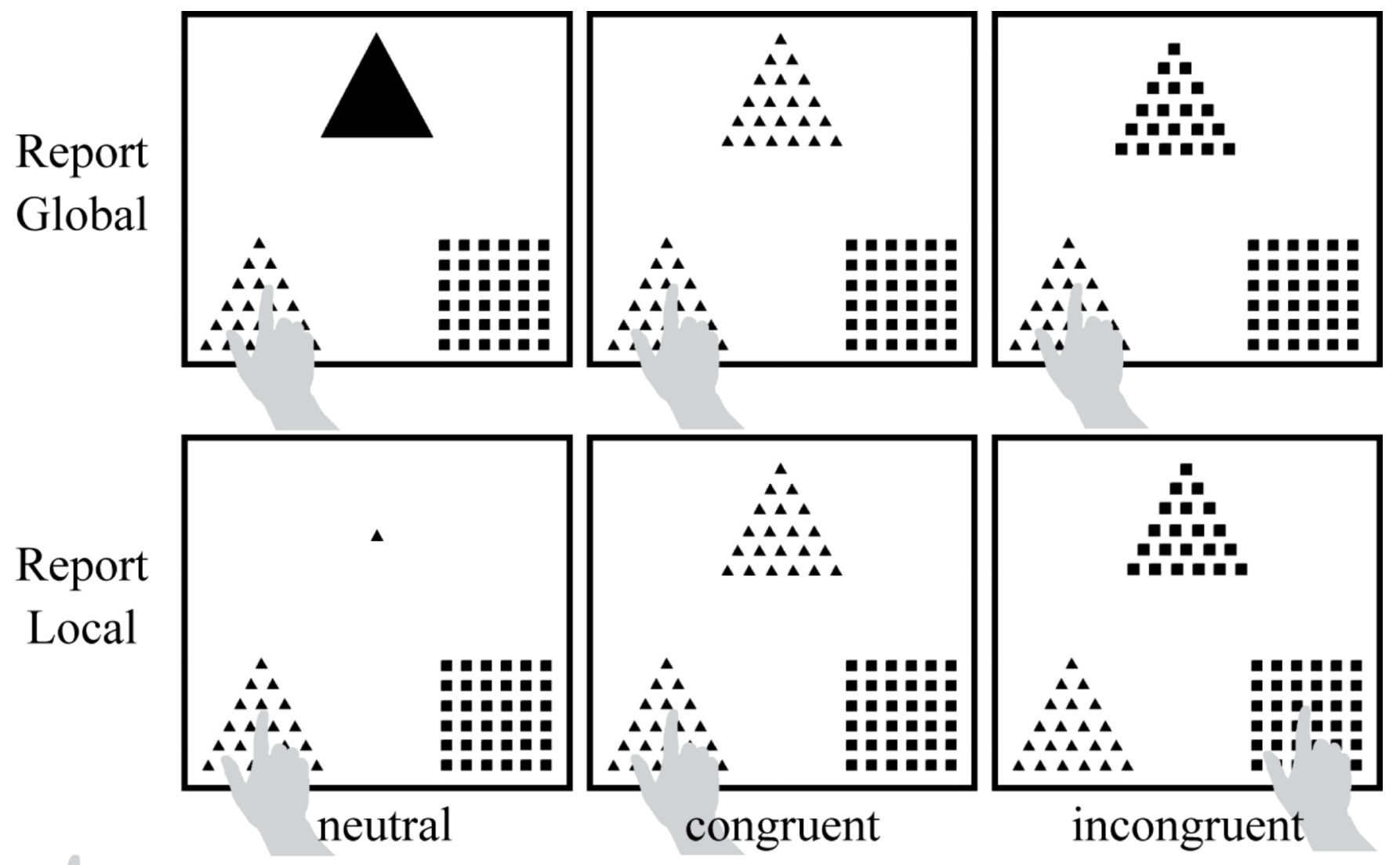

correct answer

Figure 2.

Depiction of stimuli and trial procedure for experiment 2. 


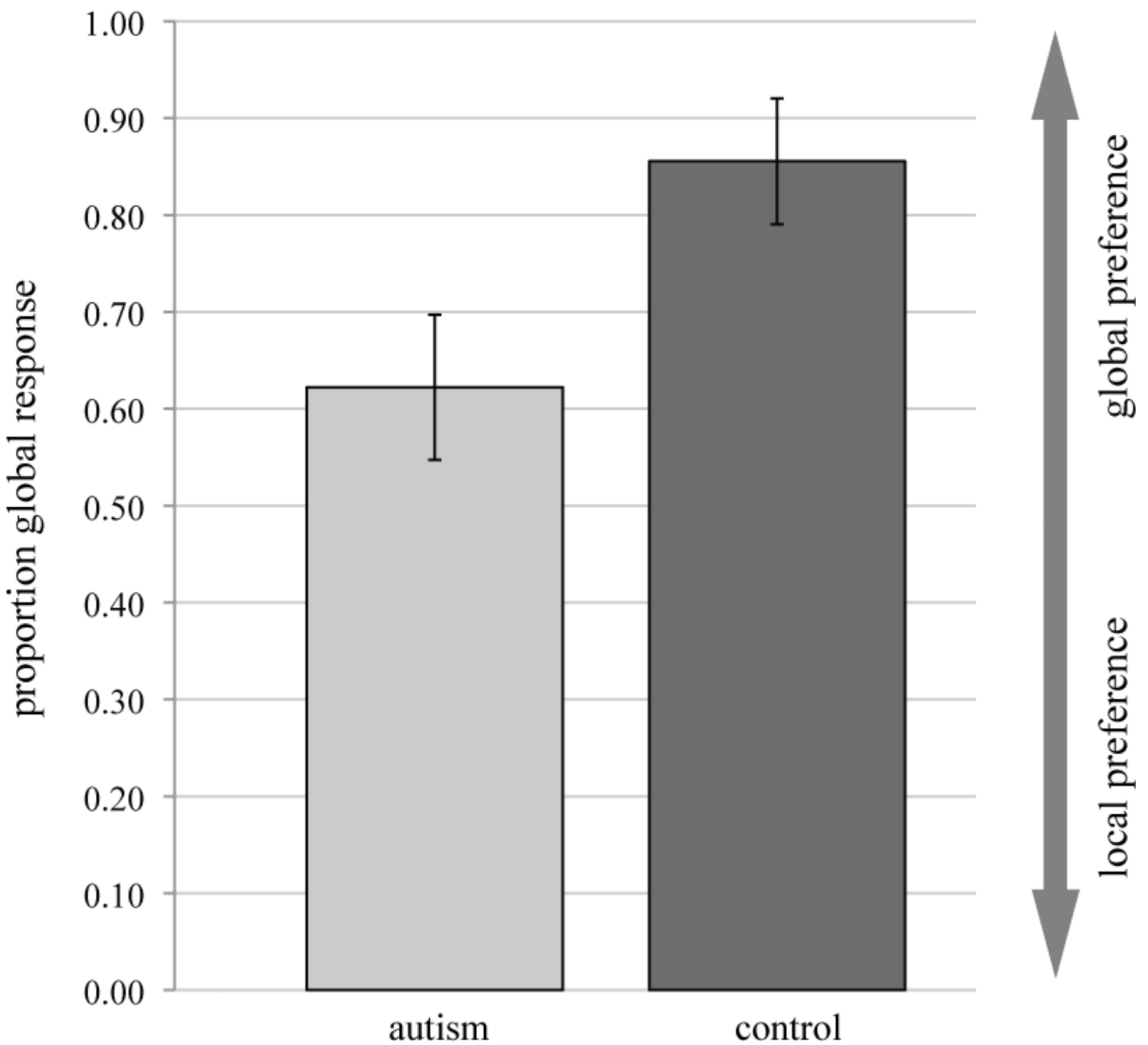

Figure 3.

Mean proportion of trials on which participants reported the global aspect of the compound stimuli in Experiment 1. 


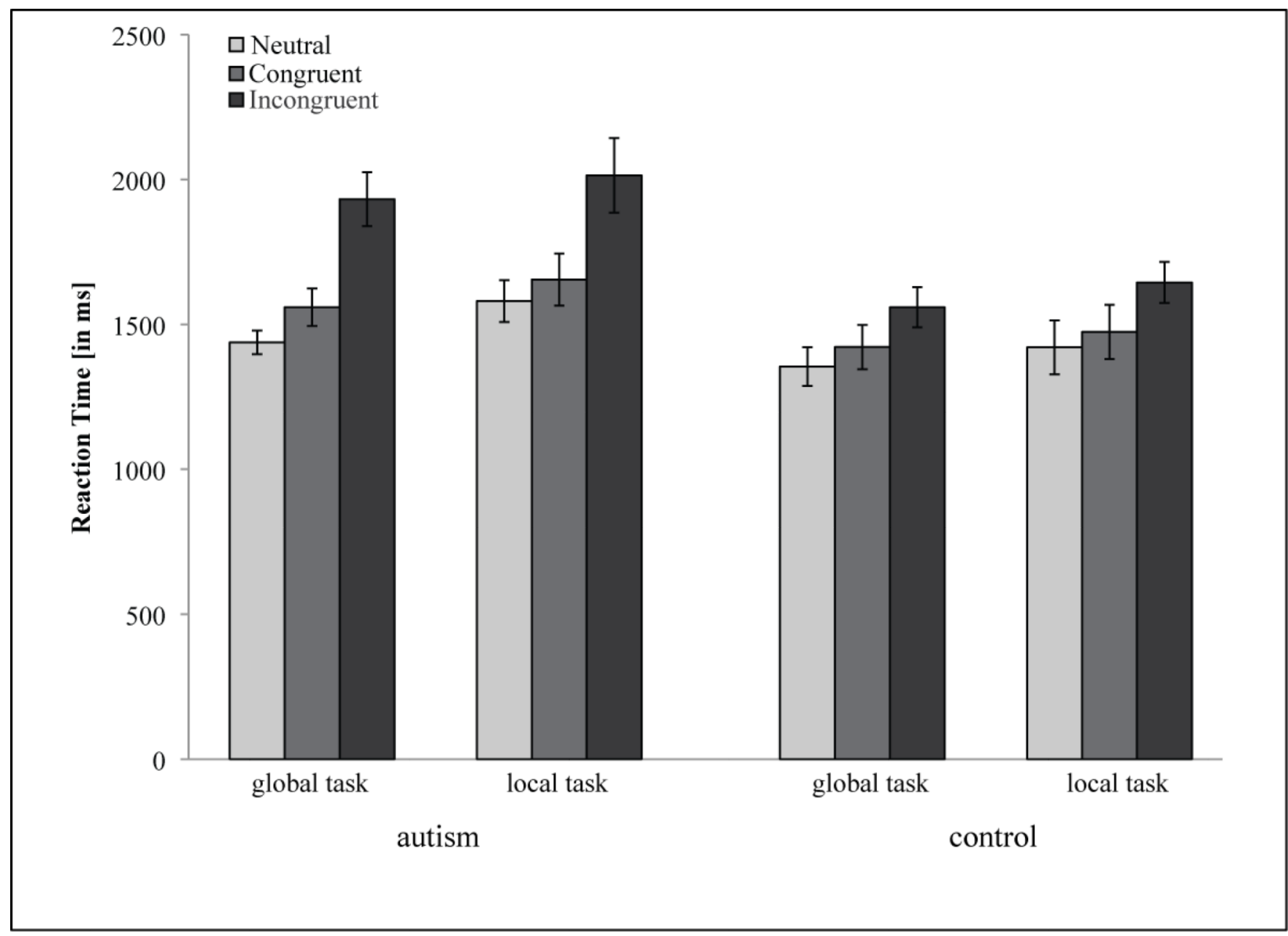

Figure 4.

Mean reaction times from Experiment 2 for neutral, congruent, and incongruent trials for both global and local tasks, separated by group. Error bars indicate standard error of the mean. 


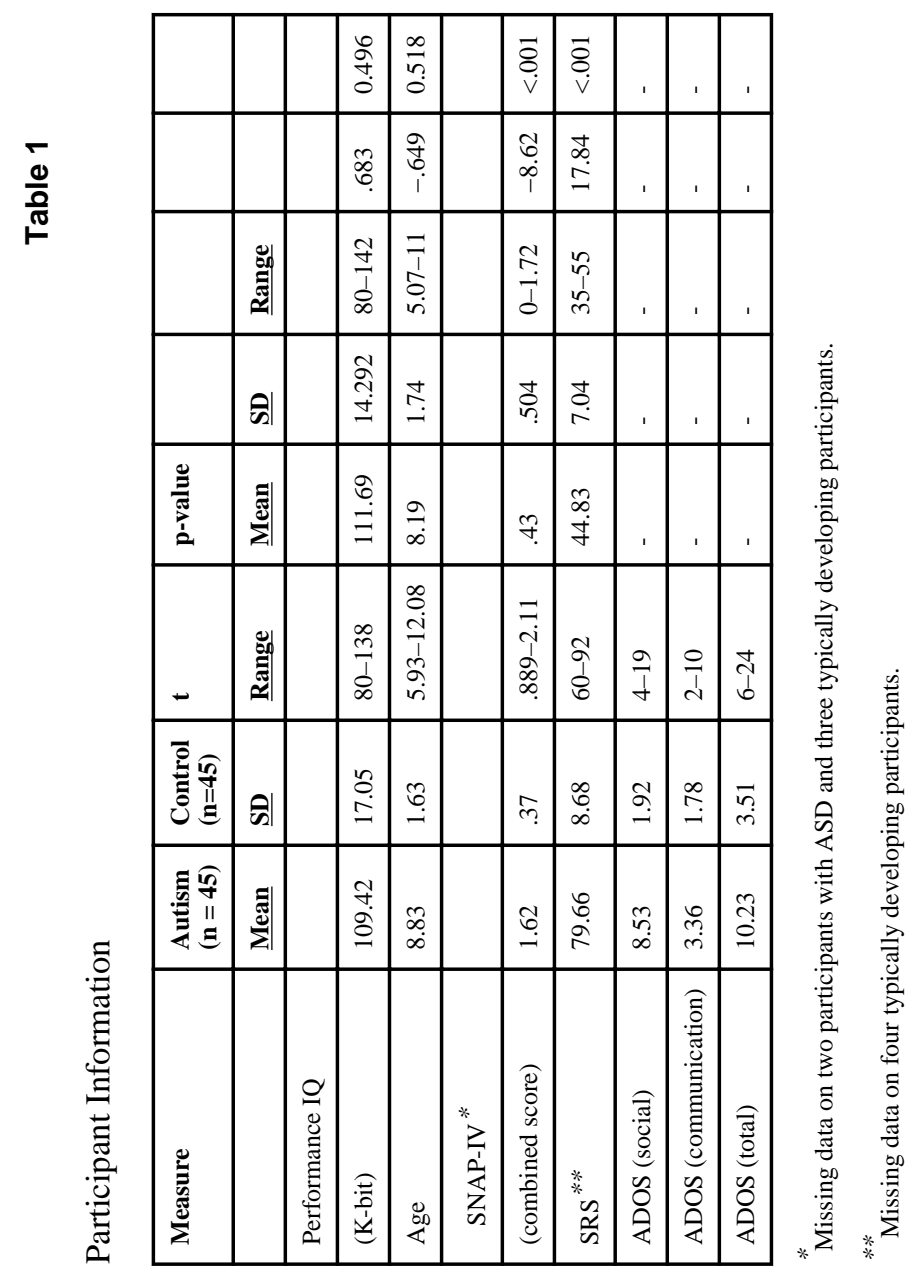

J Autism Dev Disord. Author manuscript; available in PMC 2014 October 01. 


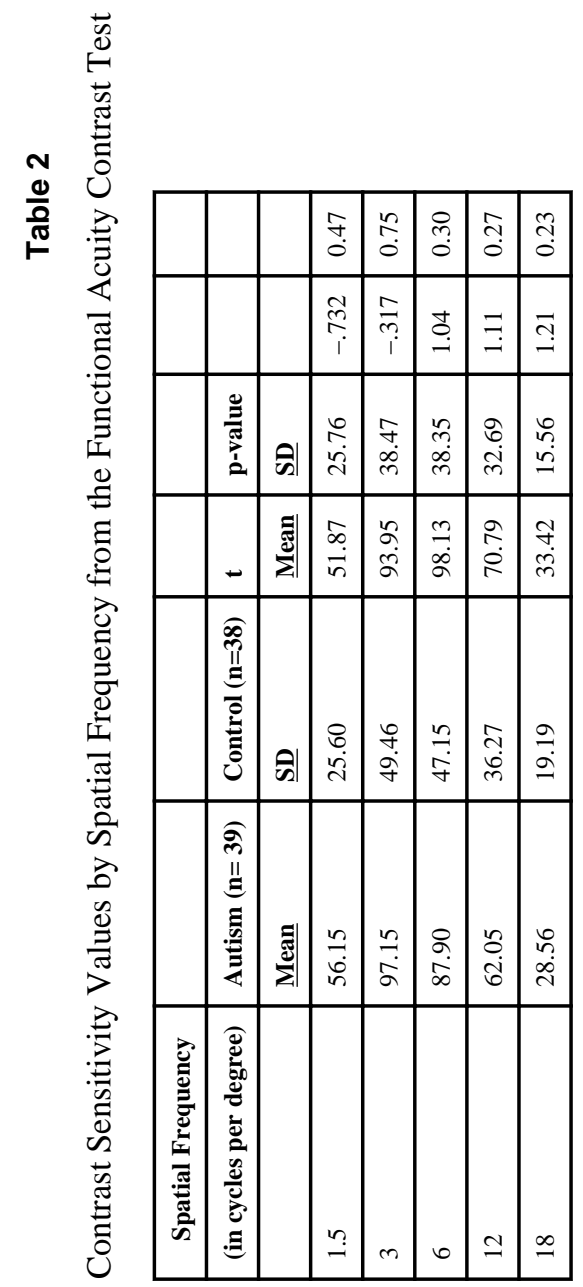




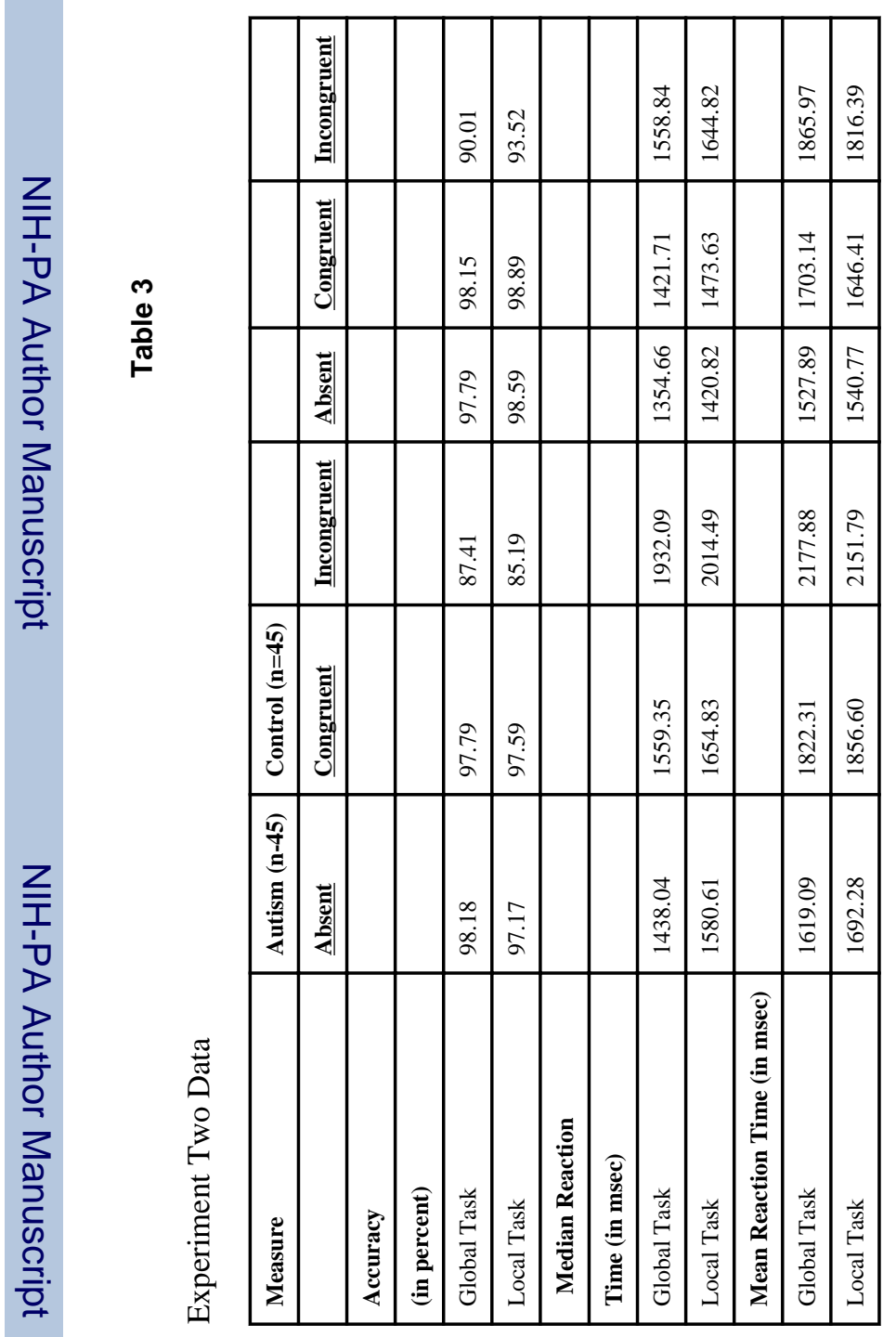

